\title{
Effect of nebivolol on fracture healing: An experimental rat model
}

\author{
Hasan Metineren ${ }^{1, A, D-F}$, Turan Cihan Dülgeroğlu ${ }^{1, A-C}$, Mehmet Hüseyin Metineren ${ }^{2, A, C}$, Ekrem Aydın ${ }^{1, A-C}$ \\ ${ }^{1}$ Department of Orthopedics and Traumatology, Medical Faculty, Dumlupınar University, Turkey \\ ${ }^{2}$ Department of Pathology, Medical Faculty, Dumlupınar University, Turkey \\ A - research concept and design; $\mathrm{B}$ - collection and/or assembly of data; $\mathrm{C}$ - data analysis and interpretation; \\ $D$ - writing the article; $E$ - critical revision of the article; $F$ - final approval of article
}

\section{Address for correspondence \\ Hasan Metineren}

E-mail:drhmetin19@gmail.com

\section{Funding sources}

None declared

\section{Conflict of interest}

None declared

Received on July 10, 2016

Revised on September 7, 2016

Accepted on 0ctober 24, 2016
DOI

$10.17219 /$ acem/66291

\section{Copyright}

Copyright by Author(s)

This is an article distributed under the terms of the

Creative Commons Attribution Non-Commercial License

(http://creativecommons.org/licenses/by-nc-nd/4.0/)

\section{Abstract}

Background. Bone metabolism is a complex system, and fracture healing is one of its most important functions. Many circumstances can influence this process. Chronic drug use in elderly populations can affect bone healing, and inadequate tissue perfusion, increased free radicals and adverse drug effects can negatively influence fracture healing. Nebivolol, an anti-hypertensive drug that selectively blocks $\beta 1$ receptors, effectively reduces blood pressure by inducing peripheral vasodilation. Nebivolol also exerts anti-oxidant effects by stimulating nitric oxide (NO) synthesis. Many studies show that NO protects the vascular endothelium and improves fracture healing.

Objectives. In this study, the histological and radiological effects of intraperitoneally administered nebivolol on fracture healing were evaluated.

Material and methods. Twenty-one Sprague Dawley rats were divided into 3 (nebivolol 1,2 and control) groups. Sterile nebivolol solution $(1 \mathrm{~mL}=0.017 \mathrm{mg}$ nebivolol) was given to the rats in group 1 every day for 4 weeks, while the rats in nebivolol group 2 were given $2 \mathrm{~mL}$ per day, beginning after the production of an open, displaced unilateral femur fracture. Radiographic and histological studies were used to evaluate fracture healing.

Results. Histological and immunohistochemical analysis showed osseous healing with woven bone at the fracture site and only minimal amounts of cartilage in nebivolol 1 and 2 groups. Radiological grading was not different between the control and the nebivolol groups.

Conclusions. This study suggests that nebivolol, a selective $\beta$ blocker, has positive effects on fracture healing through anti-oxidative effects via the NO pathway and direct vasodilator effects.

Key words: experimental model, nitric oxide, fracture healing, nebivolol 
Fracture healing is a complex process that requires a local hematoma rich in mesenchymal cells and cytokines. In the fracture healing phase, there is a strong correlation between angiogenesis and osteogenesis. Transportation of biomaterials through the blood to the fracture site is necessary for adequate and prompt healing. The process of converting the organized hematoma to bone callus includes endochondral and intramembranous ossification stages. In addition, chondrocyte differentiation and mineralization require adequate nutrition from the vascular bed. ${ }^{1-3}$

Nebivolol is a highly selective $\beta$ 1-adrenergic receptor antagonist that is used for antihypertensive therapy. 4 In many individuals, a dose of $5 \mathrm{mg}$ effectively reduces blood pressure over a $24 \mathrm{~h}$ period and has been shown to cause endothelium-dependent vasodilation. ${ }^{5,6}$ Nebivolol demonstrates systemic anti-oxidant effects by stimulating nitric oxide (NO) release. ${ }^{7} \mathrm{NO}$ is produced by endothelial NO synthase (eNOS) from L-arginine. ${ }^{6,8}$ The l-nebivolol enantiomer stimulates NO release, and the d-nebivolol enantiomer is responsible for a $\beta 1$-selective blockade.

The aim of this study was to evaluate the effect of intraperitoneally administered nebivolol on fracture healing. Histological and radiological evaluation of healing was performed in nebivolol and control groups using a rat open femoral fracture model.

\section{Material and methods}

Twenty-one adult Sprague Dawley rats (average weight $250 \mathrm{~g}$ ) were used in this study. All in vivo study protocols were approved by the Institutional Laboratory Animal Care and Use Ethical Committee. The animals were housed in the Laboratory Animal Care-Augmentation Facility of Dumlupinar University in a temperaturecontrolled room (room temperature $20-22^{\circ} \mathrm{C}$ ) on a $12 \mathrm{~h}$ light-dark cycle and were provided with rat pellets with water ad libitum. There were 7 animals in each standard cage.

\section{Nebivolol preparation}

Five-milligram nebivolol tablets (Vasoxen, Menarini Group, I. E. Ulagay, Germany) were pulverized and dissolved in distilled water to obtain a $0.017 \mathrm{mg} / \mathrm{mL}$ solution. This was equivalent to a $5 \mathrm{mg}$ adult human $(70 \mathrm{~kg})$ dose.

\section{Surgical procedure}

The 21 rats were randomly divided into 3 groups: nebivolol 1, nebivolol 2, and the control. Following the induction of anesthesia with intraperitoneal injection of ketamine $(50 \mathrm{mg} / \mathrm{kg})$ and xylazine hydrochloride $(10 \mathrm{mg} / \mathrm{kg})$, the right hind limb of each animal was shaved and pre- pared with chlorhexidine gluconate for aseptic surgery. Using an aseptic technique, a longitudinal incision was made on the lateral aspect of the right hind limb. An open femoral fracture was created on the midshaft of the femur using a $1.0 \mathrm{~mm}$ sterile drill (Aysam Samsun, Turkey). Two perpendicular drill holes were made in the middle of the shaft, and the bone was manually broken. An intramedullary fixation was performed using a $1.50 \mathrm{~mm}$ (0.057 inch)-diameter stainless Kirschner wire (Aysam Samsun, Turkey). The wound was closed using 5-0 vicryl sutures (Pegelac, Doğsan Trabzon, Turkey), and the skin was closed with polypropylene 4-0 sutures (Propylene, Doğsan Trabzon, Turkey). The rats were left without cast immobilization for 4 weeks (Fig. 1).

\section{Nebivolol treatment}

Sterile nebivolol solution $(1 \mathrm{~mL})$ was given to the rats in group 1 every day for 4 weeks, while the rats in nebivolol group 2 were given $2 \mathrm{~mL}$ per day. At the end of the $4^{\text {th }}$ week, all rats were sacrificed, and the broken femurs were removed. Radiological examinations were first performed before fixing the femurs for 2 days in $10 \%$ buffered formaldehyde solution for histological examination.

\section{Histological and immunohistochemical examination}

The specimens were decalcified before embedding in paraffin. They were then sectioned $(4 \mu \mathrm{m})$, placed on slides, and stained with hematoxylin \& eosin (H\&E) and Masson's trichrome. CD34 immunostaining was used to detect angiogenesis. Each specimen was graded based on 10 sections. Grades were determined based on the visual field. Slides were examined using a light microscope (Olympus BX51, Tokyo, Japan) for fracture healing and new bone formation by pathologists who were blinded to the groups. The histological assessment scale (Table 1) described by Allen et al. was used for this study. ${ }^{9}$

Table 1. Histological fracture healing scale

\begin{tabular}{|l|c|}
\multicolumn{1}{|c|}{ Histological grade } & Score \\
\hline Pseudoarthrosis & 0 \\
\hline Incomplete cartilage union & 1 \\
\hline Complete cartilage union & 2 \\
\hline Incomplete bone union & 3 \\
\hline Complete bone union & 4 \\
\hline
\end{tabular}


Fig. 1. Surgical procedure; open femoral fracture model and pinning

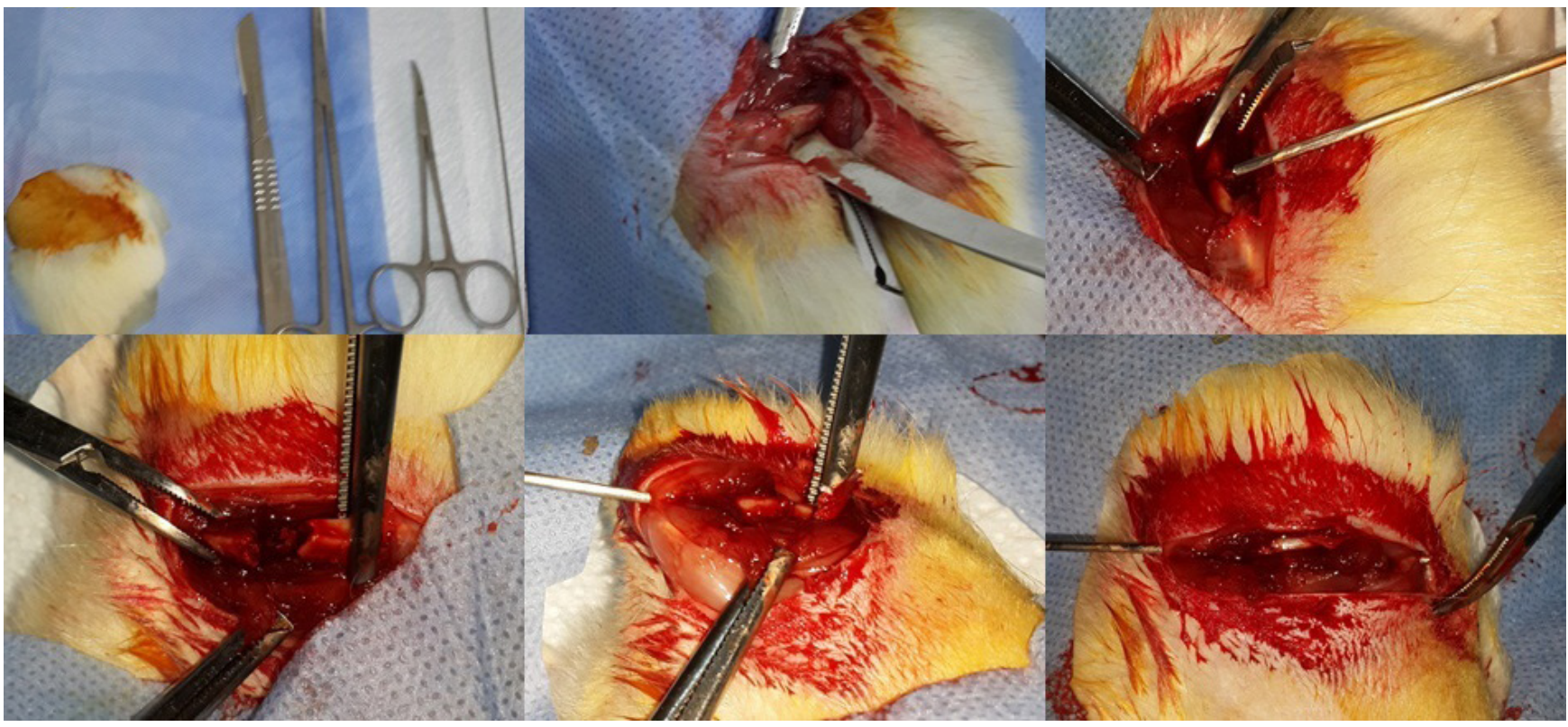

Fig. 2. Control group histological sections: a) Masson's trichrome staining showed large amounts of cartilage (yellow arrow) in the callus; b) No new vascular bed was shown with CD34 immunostaining; c) Minimally trabecular bone visualized with hematoxylin \& eosin (H\&E) staining ( $\times 40)$

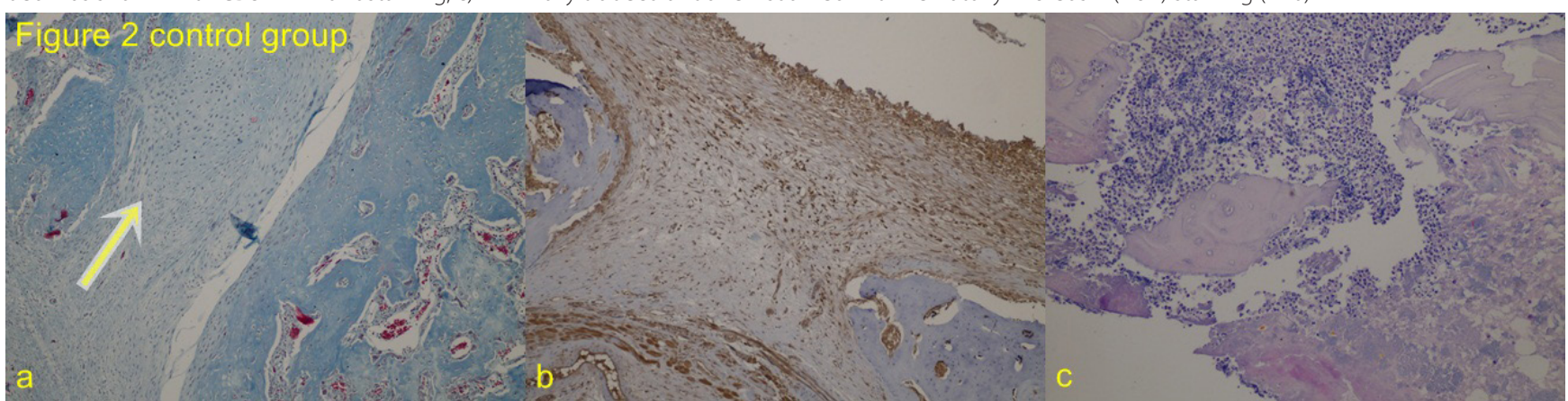

\section{Radiological examination}

Standard anteroposterior roentgenograms were taken. An orthopedic surgeon evaluated the radiographic data according to the scale. Fracture healing was radiographically evaluated and graded into Class 0 (non-union), Class I (mild union), and Class II (union) groups. ${ }^{10}$

\section{Statistical analysis}

Radiologic and histologic scores were determined at the end of the $4^{\text {th }}$ week. Statistical analyses were performed using GraphPad Prism v. 6.05 (GraphPad Software, Inc., La Jolla, USA). All data was expressed as mean \pm standard deviation (SD). Because of the small experimental groups, we used nonparametric statistical tests. The differences among the multiple groups were analyzed with the Kruskal-Wallis test. The differences between 2 groups were analyzed with Dunn's post-hoc test. A p-value $<0.05$ was considered as statistically significant.

\section{Results}

All rats completed the 4-week study. K-wire migration was detected in the abdominal region of one rat in nebivolol group 1; the rat was not excluded from the study because it had no negative impact on animal welfare. The histological findings are shown in Fig. 2-4.

\section{Histological findings}

The average histological scores for the control (Fig. 2), and nebivolol groups 1 and 2 (Table 2) were 1.71, 2.28, and 2.85 , respectively. Histopathological scoring results revealed that statistically significant differences were observed between the study groups $(p=0.007)$, as well as between nebivolol groups 1 and 2 ( $\mathrm{p}<0.005)$. Vascularity (red arrows) was remarkable in nebivolol group 2 (Fig. 4). Trabecular bone formation was significant in nebivolol groups 1 and 2 (Fig. 3, 4). Cartilage formation occurred at a higher rate in the control group (yellow arrow), compared with the nebivolol groups (Fig. 2). 
Fig. 3. a) Masson's trichrome stained micrographs at the fracture site; cartilage formation on the left side and trabecular bone on the right; b) CD34 immunostaining showed mild vascularity; c) Hematoxylin \& eosin staining showed increased trabecular bone formation in the nebivolol group 1 ( $\times 40$ )

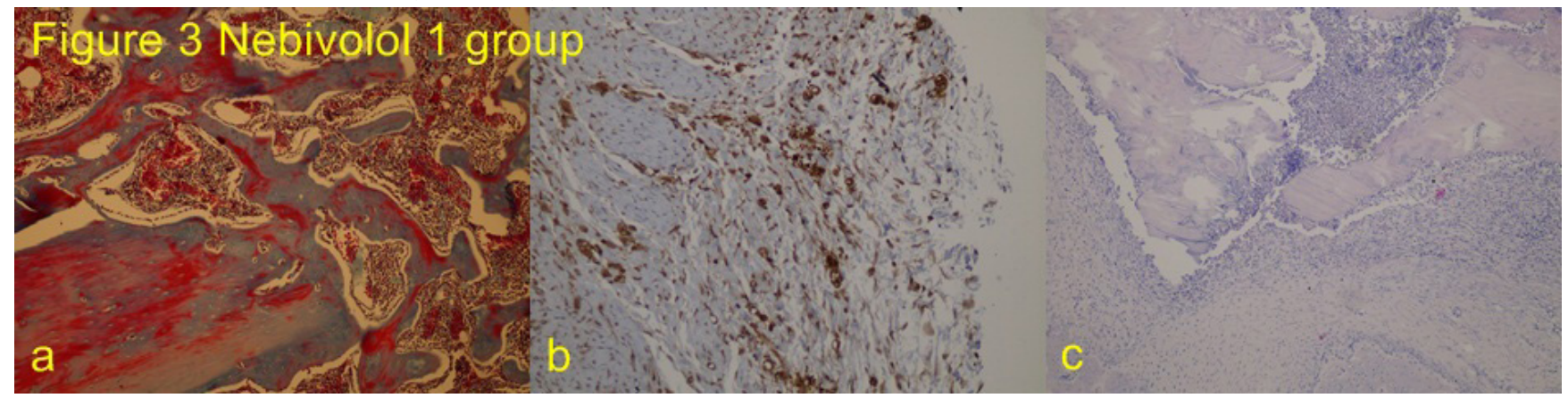

Fig. 4. a) Masson's trichrome and c) hematoxylin \& eosin micrographs showed predominantly trabecular bone and less cartilage formation; b) CD34 immunostained micrographs showed increased vascularity in nebivolol group $2(\times 40)$

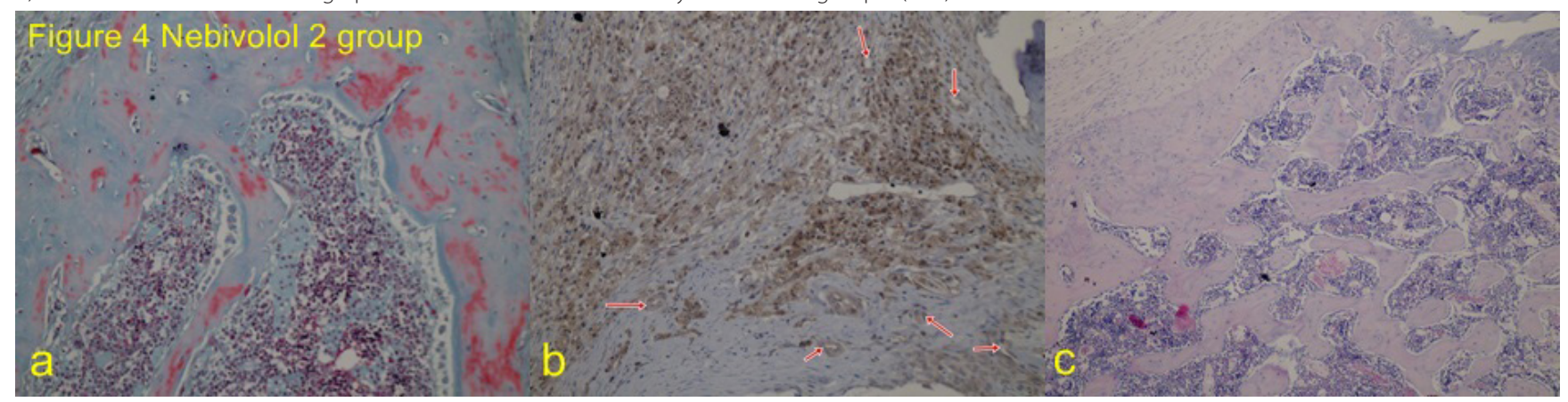

Fig. 5. Radiological findings of the 3 groups

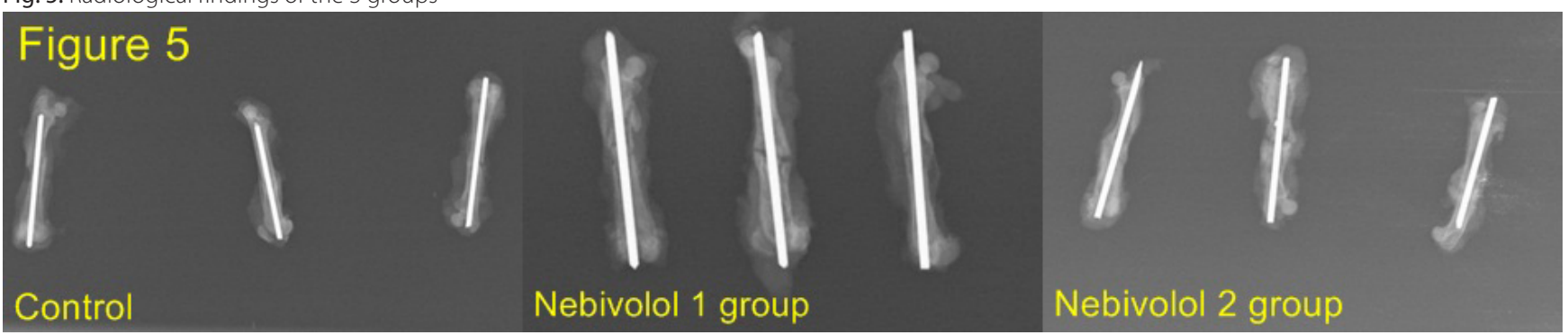

Table 2. Statistical evaluation of histologic and radiological scores

\begin{tabular}{|l|c|c|c|c|}
\hline Parameters & Control & Nebivolol 1 & Nebivolol 2 & p-value \\
\hline $\begin{array}{l}\text { Histological } \\
\text { score }\end{array}$ & $1.71 \pm 0.75 a$ & $2.28 \pm 0.75$ & $2.85 \pm 0.78 \mathrm{a}$ & 0.007 \\
$\begin{array}{l}\text { Radiological } \\
\text { score }\end{array}$ & $1.14 \pm 0.69$ & $1.28 \pm 0.53$ & $1.71 \pm 0.27$ & 0.088 \\
\hline
\end{tabular}

Data are presented as mean \pm standard deviation (SD); data were tested using the Kruskal-Wallis test and the Dunn's method was used for post hoc testing; $p$-value < 0.05 was considered statistically significant; $\mathrm{p}^{\mathrm{a}}<0.05$, compared to the control group.

\section{Radiological findings}

The average radiological scores were 1.14, 1.28, and 1.71 in the control and nebivolol groups 1 and 2, respectively. There were no statistically significant differences in radiographic results between the 3 groups and 2 groups (Fig. 5).

\section{Discussion}

Hypertension and osteoporosis that results in poorly healing fractures are frequently seen in elderly populations. In addition, elderly populations typically have higher and more continuous drug use, due to the presence of chronic conditions. Chronic drug use can positively or negatively affect bone metabolism. Heparin and its derivatives have negative effects on the bone microstructure, while angiotensin-converting enzyme inhibitors can reduce fracture risk. ${ }^{11,12}$ The selective $\beta 1$-blocker nebivolol, which is frequently used for hypertension, has both vasodilation and anti-oxidation effects. Recent studies suggest that high concentrations of nebivolol result in healing through the increased release of NO. ${ }^{13}$ Gülcan et al. and Schaffer et al. previously showed that nebivolol had positive effects on wound healing. ${ }^{14,15}$ However, according to our knowledge, this study is the first to investigate the effects of nebivolol on fracture healing. 
The histochemical results of this study clearly show the positive effects of nebivolol on fracture healing. Complete bone union and trabecular bone formation were significantly higher in nebivolol group 2 (0.007) (Fig. 4). There were significant differences between the $5 \mathrm{mg} / \mathrm{kg}$ (nebivolol 1) treatment and the $10 \mathrm{mg} / \mathrm{kg}$ (nebivolol 2) treatment groups ( $\mathrm{p}<0.005)$. This suggests that nebivolol has a dose-dependent effect on healing. $\beta 1$-receptors cause vascular endothelial injury, and nebivolol protects endothelial cells exerting anti-oxidative effects and prevents atherosclerosis. ${ }^{16}$ However, nebivolol can also prevent osteoporosis by selectively inhibiting sympathetic nervous system $\beta 1$ adrenergic receptors. ${ }^{17}$ Nebivolol inhibits NO synthase uncoupling and produces systemic antioxidant effects. Thus, the beneficial activity of nebivolol is attributed to both the inhibition of eNOS uncoupling and endogenous antioxidant properties that lead to free-radical scavenging. ${ }^{6,18}$ Interestingly, nebivolol is the only selective $\beta 1$-blocker that has anti-oxidative properties. ${ }^{16}$ Turner et al. showed that treatment with an NOS inhibitor decreased new bone formation in a rat tibia model. ${ }^{19}$ In addition, supplementation with NO induces new bone formation, and NO-mediated vasodilation increases blood flow during the early phases of fracture healing. ${ }^{7,20}$

There was no significant difference in radiographic results; however, nebivolol has a significantly positive effect on bone microstructure healing by inducing NO release. Therefore, the histological results obtained in this study could not be confirmed radiologically.

The results of this study suggest that nebivolol has positive effects on bone healing and thus can be used to treat hypertension in elderly populations. The limitations of the present study include the small sample size, which was a result of ethical considerations. In addition, bone healing was not determined through micro-computed tomography or positron emission tomography due to cost restrictions. These additional tests may demonstrate the vasodilatory effects of nebivolol for further support of our findings.

\section{Conclusions}

This study suggests that nebivolol, a selective $\beta$ blocker, has positive effects on fracture healing through anti-oxidative effects via the NO pathway and direct vasodilator effects.

\section{References}

1. McKibbin B. The biology of fracture healing in long bones. $J$ Bone Joint Surg Br. 1978;60:150-162.

2. Cornell CN, Lane JM. Newest factors in fracture healing. Clin Orthop Related Res. 1992;277:297-311.

3. Einhorn TA, Majeska RJ, Rush EB, Levine PM, Horowitz MC. The expression of cytokine activity by fracture callus. J Bone Miner Res. 1995;10:1272-1281.
4. Xhonneux R, Wouters L, Reneman RS, Janssen PA. The I-enantiomer of nebivolol potentiates the blood pressure lowering effect of the d-enantiomer. Eur J Pharmacol. 1990;181:261-265.

5. Broeders MA, Doevendans PA, Bekkers BC, et al. Nebivolol: A thirdgeneration beta-blocker that augments vascular nitric oxide release: Endothelial beta-2 adrenergic receptor-mediated nitric oxide production. Circulation. 2000;102:677-684.

6. Troost R, Schwedhelm E, Rojczyk S, Tsikas D, Frölich JC. Nebivolol decreases systemic oxidative stress in healthy volunteers. $\mathrm{Br} J \mathrm{Clin}$ Pharmacol. 2000;50:377-379.

7. Tomlinson RE, Shoghi KI, Silva MJ. Nitric oxide-mediated vasodilation increases blood flow during the early stages of stress fracture healing. J Appl Physiol. 2014;15(116):416-424.

8. Allen HL, Wase A, Bear WT. Indomethacin and aspirin: Effect of nonsteroidal anti-inflammatory agents on the rate of fracture repair in the rat. Acta Orthop Scand. 1980;51:595-600.

9. Goldberg VM, Powell A, Shaffer JW, Zika J, Bos GD, Heiple KG. Bone grafting: Role of histocompatibility in transplantation. J Orthop Res. 1985;3:389-404.

10. Xia J, Zhang Z, Wang J, Zu J, Wang N, Wang D. Comparison of the effects of heparin and the direct factor Xa inhibitor, rivaroxaban, on bone microstructure and metabolism in adult rats. Connect Tissue Res. 2015;56:477-482.

11. Nakagami H, Osako MK, Morishita R. Potential effect of angiotensin II receptor blockade in adipose tissue and bone. Curr Pharm Des. 2013;19:3049-3053.

12. Gao Y, Nagao T, Bond RA, Janssens WJ, Vanhoutte PM. Nebivolol induces endothelium-dependent relaxations of canine coronary arteries. J Cardiovasc Pharmacol. 1991;17:964-969.

13. Gülcan E, Kuçuk A, Cayci K, et al. Topical effects of nebivolol on wounds in diabetic rats. Eur J Pharm Sci. 2012;47:451-455.

14. Schaffer MR, Tantry U, Gross SS, et al. Nitric oxide regulates wound healing. J Surg Res. 1996;63:237-240.

15. Mason RP, Kubant R, Jacob RF. Effect of nebivolol on endothelial nitric oxide and peroxynitrite release in hypertensive animals: Role of antioxidant activity. J Cardiovasc Pharmacol. 2006;48:862-869.

16. Pettersson K, Bejne B, Björk H, Strawn WB, Bondjers G. Experimental sympathetic activation causes endothelial injury in the rabbit thoracic aorta via beta 1-adrenoceptor activation. Circ Res. 1990;67:1027-1034.

17. Fedorchenko IL, Nebieridze DV, Safarian AS, Skripnikova IA, Sobchenko KE. Cardiovascular diseases and osteoporosis: What is common? Ter Arkh. 2014;86:116-119.

18. Mollnau H, Schulz E, Daiber A, et al. Nebivolol prevents vascular NOS III uncoupling in experimental hyperlipidemia and inhibits NADPH oxidase activity in inflammatory cells. Arterioscler Thromb Vasc Biol. 2003;23:615-621.

19. Turner $\mathrm{CH}$, Takano Y, Owan I, Murrell GA. Nitric oxide inhibitor L-NAME suppresses mechanically induced bone formation in rats. Am J Physiol. 1996;270:634-639.

20. Diwan AD, Wang MX, Jang D, Zhu W, Murrell GA. Nitric oxide modulates fracture healing. J Bone Miner Res. 2000;15:342-351. 